Fecha de recepción: febrero 2019 Fecha de aceptación: julio 2019 Versión final: octubre 2020

\section{Propuesta de categorización de habilidades en estudiantes y profesionales noveles de Diseño}

León Felipe Irigoyen Morales*

Resumen: Después de revisar antecedentes relacionados con la valoración de competencias, se propone un esquema que ubique en categorías claramente definidas el amplio repertorio de habilidades asociadas con la formación profesional de un diseñador gráfico. La intención principal es organizar competencias específicas que permitan distintos niveles de desarrollo. La propuesta puede ser empleada de forma cotidiana o bien, en proyectos institucionales de rediseño de planes de estudio o ajuste curricular ya que permite visualizar con relativa facilidad el impacto y la relevancia de múltiples actividades académicas típicamente asociadas con el Diseño Gráfico.

Palabras clave: Diseño - habilidades - competencias - educación superior - profesión.

[Resúmenes en inglés y portugués en las páginas 140-141]

${ }^{(*)}$ Profesor-investigador de Tiempo Completo del programa en Diseño Gráfico y presidente de la Academia de Investigación e Innovación en Diseño, de la Universidad de Sonora (Hermosillo, México). Licenciado en Diseño Gráfico por la Universidad Gestalt de Diseño (Xalapa, Ver.) y Maestro en Diseño Gráfico Digital por la Universidad Iberoamericana (Tijuana, B.C.). Desde el 2011 se ha desempeñado como catedrático en áreas de tecnología e innovación.

\title{
Introducción
}

Desde hace aproximadamente cuatro décadas, la educación en distintos niveles, así como su misma evaluación objetiva, se ha estado replanteando a nivel internacional con un Enfoque Basado en Competencias (EBC); y con ello, se han logrado transformar sistemas educativos nacionales, modelos curriculares y planes de estudios institucionales específicos.

La tarea emprendida por expertos en la materia, así como por secretarías y ministerios de educación en múltiples países no es nada fácil, y seguirá requiriendo de una inversión de recursos enorme hasta que se demuestren los resultados de esta macro tendencia. Esta iniciativa se complica aún más porque se utilizan distintas acepciones de la terminología relacionada y las múltiples perspectivas sólo coinciden en la posibilidad de movilizar e integrar diversos saberes cuando el profesionista entra en contacto con situaciones únicas que requieren del empleo de sus capacidades de resolución de problemas (DGESPE, s/f). 
Dicho enfoque, se orienta a la posibilidad de utilizar no sólo una serie definida de conocimientos y habilidades que permitan a los estudiantes cumplir un objetivo, sino que suman e integran de manera natural otras características necesarias para la solución de problemas particulares de forma eficiente, racional y ética.

Estos cambios se han implementado a nivel mundial, con distintos niveles de penetración y grados de aceptación, debido a numerosas circunstancias de tipo económico y político. Aunado a que las iniciativas, aunque relativamente bien intencionadas, se han topado con problemáticas específicas de carácter social y cultural en distintas latitudes, que dificultan e incluso cuestionan el "abandono" del enfoque por objetivos, en pro del enfoque centrado en competencias.

Sin embargo, las esferas en las que se toman muchas de las decisiones y se estudia el tema, son totalmente independientes a las posibles opiniones de la gran mayoría de los afectados, reduciendo el margen de acción de los profesores promedio. No obstante, existe una preocupación generalizada al momento de la aplicación específica, ya que son pocos los estudios o las aplicaciones disciplinares concretas que, en primer lugar, puedan llegar a acuerdos entre sus promotores y detractores, sin afectar el funcionamiento cotidiano de las escuelas; y después, que sean capaces de homologar tanto los conocimientos como las competencias indispensables que, en teoría, responden a una realidad económica y laboral actualizada.

En el caso de las ciencias o las ingenierías, es relativamente posible delimitar el universo de conocimiento y habilidades que buscan generarse en un estudiante universitario, ya sea por medio de un enfoque académico o de una especificación tal, delimitada por el nivel de complejidad deseado.

Pero, en el caso de las disciplinas como el Diseño, que no poseen un rigor temático similar o un perfil de egreso consensado (muchas veces definido por asociaciones o colegios), resulta mucho más complejo hablar de las competencias necesarias de un profesionista; y es cuando iniciamos con la discusión del perfil deseable de nuestros estudiantes.

Ahora bien, cabe señalar que las características específicas del tipo de escuela (privada o pública), la disponibilidad y penetración tecnológica, la sensibilidad cultural hacia nuevas tendencias y el acceso a recursos económicos (que varía ampliamente entre países), así como otras circunstancias administrativas u organizacionales (como el departamento o facultad a las que pertenecen los programas); afectan directamente el tipo de egresado universitario y del profesionista de Diseño. Otros aspectos, como la duración de los programas, la variedad o la importancia de las industrias regionales de cada latitud, o la demanda específica que este tipo de profesionistas tiene en el tiempo, modifica la percepción general que se tiene del diseñador.

Cabe mencionar que, en ocasiones, se genera una discusión entre académicos, respecto a la relevancia que tiene el hecho de que los estudiantes de Diseño a nivel licenciatura sean capacitados para investigar tal como lo haría un estudiante de ciencias. Esto resulta atractivo y es promovido por profesores que adicionalmente cumplen funciones de investigadores, quienes buscan generar otro tipo de competencias relacionadas con el desarrollo de ideas complejas, la innovación, así como la vinculación entre sistemas y disciplinas. Sin embargo, cuando se mantiene un esquema tradicional de simple transferencia de información, no se generarán este tipo de habilidades. 
Entonces, para hablar de las necesidades específicas que la enseñanza, el estudio e incluso la investigación del Diseño requieren, es necesario plantear escenarios y planes de estudio que, debido a la naturaleza misma de la disciplina, no pueden considerarse nunca como verdaderamente actualizados, pero sí pueden poseer una flexibilidad tal que permita ajustar o modificar fácilmente la inclusión o el remplazo de conocimientos específicos dentro de la misma malla curricular.

Por otro lado, el Diseño siempre ha sido una disciplina difícil de definir y se le ubicará en el área temática cuya interpretación resulte más afín, en ocasiones con Facultades de Artes Plásticas, a veces con Arquitectura, e incluso, ligado a la Mercadotecnia o Ingeniería. Independientemente de estas situaciones, el establecimiento de las pautas necesarias para su enseñanza y estudio, requieren indudablemente una adaptación urgente que permita incluir constantemente componentes de innovación adaptables a una realidad social, económica y tecnológica que muta cada vez con mayor rapidez y con impactos más profundos. La propuesta planteada en este artículo va ligada directamente a la necesidad de definir claramente qué tipos de competencias se busca desarrollar en los estudiantes, qué tan estrecha es su relación con la realidad profesional en su aplicación práctica, qué tan susceptibles y resilientes somos como docentes a los cambios profundos y cuál es la disposición del programa al momento de requerir adaptaciones.

\section{Antecedentes}

Con respecto a la definición de habilidades, the North Central Regional Educational Laboratory y the Metiri Group (2003) detallan claramente cómo debe componerse el aprendizaje del siglo XXI, desde el punto de vista del logro académico. Lo dividen en cuatro grandes áreas que, aunque no están pensadas directamente para ser aplicables a un programa de Diseño, resultan sumamente pertinentes como punto de partida.

En la primera de las áreas, se ubican las habilidades denominadas como Alfabetidad de la era digital (Digital-Age Literacy) donde se encuentran algunas como: alfabetidad básica en materia científica, económica y tecnológica; alfabetidad visual y de información; $y$, conciencia global y alfabetidad multicultural. En esta primera área cabe destacar que el manejo hábil de Tecnologías de la Información y la Comunicación (TIC) ya no es opcional y, aunque el nivel de destreza de los estudiantes puede variar, es relativamente fácil establecer la pauta mínima indispensable para determinar el domino de las mismas.

El Pensamiento inventivo (Inventive Thinking) es otra de las áreas propuestas donde se encuentran tres grupos de habilidades como: adaptabilidad, manejo de la complejidad y autodirección; curiosidad, creatividad y toma de riesgos; y, pensamiento de orden superior y razonamiento sonoro. Este último, se entiende como "la serie de procesos cognitivos de análisis, comparación, inferencia e interpretación, evaluación y síntesis aplicada a un rango de dominios académicos y contextos de resolución de problemas" (NCREL y the Metiri Group, 2003, p. 33).

Haciendo un paréntesis, es importante resaltar la importancia de este tipo de competencias en el arte y el Diseño, sin embargo, aún no hemos hecho lo suficiente para saber cómo desarrollarlas ni hemos coincidido en cómo evaluarlas. Además, resulta difícil incluirlas en 
las actividades cotidianas ya que hay un componente actitudinal involucrado que depende mucho de la postura del docente que interpretará y valorará de forma distinta este tipo de actividades.

En tercer lugar, la comunicación efectiva (Effective communication) incluye otro tipo de habilidades como: trabajo en equipo, colaboración y habilidades interpersonales; responsabilidad, personal, social y cívica; y comunicación interactiva. Finalmente, la última de las áreas tiene que ver con la alta productividad (High Productivity), donde las destrezas tienen que ver con: priorización, planeación y gestión para los resultados; el uso efectivo de herramientas reales; $y$ la habilidad para producir, productos relevantes de alta calidad. En el estudio antes mencionado, NCREL y the Mitiri Group (2003), se explican a detalle cada una de las habilidades propuestas y se contrastan incluso con otras previamente expuestas en estudios similares (p. 74).

Ahora bien, con un enfoque específico en programas de Diseño Gráfico, Massaguer (2017) inicia su planteamiento señalando que "los profundos cambios sociales y económicos que están afectando los tipos de estructura laboral, se extienden a la inserción laboral de los graduados" (p. 96).

El marco en el que su estudio se plantea radica en la correspondencia de las habilidades en las que se basan los planes de estudios en el ámbito formativo y la demanda que se le hace a la profesión. Menciona también la necesidad de construir herramientas para diagnosticar el grado de ajuste entre las competencias del grado en Diseño, y las necesidades reales que el ámbito profesional requiere. Lo ideal sería poder llegar a actualizar, de manera periódica, las competencias definidas por el perfil académico, e incluso, proponer nuevas vías profesionales dentro del sector.

Massaguer (2017) utiliza una escuela en particular como caso de estudio (EINA Centre Universitari de Disseny i Art de Barcelona) y los datos son obtenidos de forma directa para su análisis profundo. Sin embargo, en esta ocasión lo que resulta pertinente retomar son precisamente las habilidades y la clasificación empleada: Competencias específicas y Competencias transversales.

En el primer grupo, se mencionan un total de 28 competencias de las cuales se destacan algunas que, para esta propuesta, son de mayor interés. De inicio, se busca que todas estas competencias puedan ser fácilmente identificables, objetivamente verificables y que, en todo caso, permitan distintos niveles de profundidad. Entre las que no se seleccionaron se encuentran algunas que resultan ser demasiado vagas (detección de problemas de Diseño, uso adecuado del dibujo, uso adecuado de la tipografía y la maquetación de una página), para realzar aquellas que precisan la acción a tomar y la forma en cómo lograrlo. Finalmente, al momento de establecer la competencia y la forma en cómo se redacta, se explicita el objetivo a lograr.

Destacan algunas habilidades claramente definidas tales como: evaluación de la viabilidad (tecnológica, económica y del entorno social al que van destinados) de los proyectos de Diseño; exposición y razonamiento oral (y escrito) de los proyectos de Diseño; valoración crítica de proyectos de Diseño (tanto propios como ajenos); y otras que pueden apreciarse como altamente complejas, pero que se definen de forma adecuada: conocimiento sobre el marco legal que envuelve las actividades de Diseño; aplicación de criterios ergonómicos, y parámetros antropométricos y perceptuales, acordes a las características de uso del 
proyecto; comprensión del entorno económico y empresarial donde se desenvolverá el proyecto; entre otras.

Asimismo, se identificó un tercer grupo de competencias relacionadas con aspectos más difíciles de verificar (seguridad en el trazo y la realización de bocetos rápidos; conocimiento de instituciones y asociaciones que conforman el mundo profesional del Diseño; uso intencional de recursos gráficos para sintetizar y mejorar la comunicación) que inicialmente requieren el establecimiento de normas consensuadas, o bien, al menos de una instrumentación clara para poder comprobar si el alumno posee, o no, esa habilidad.

Finalmente, Massaguer (2017) propone otras competencias complementarias, designadas como Transversales, donde el nivel de conocimiento y experiencia requerido es mucho mayor, tanto en la complejidad de la tarea como en la comprobación de la misma. Entre ellas se encuentran ejemplos como: elaboración de informes profesionales; atención a los cambios que se van produciendo; uso de la creatividad en contextos y situaciones diferentes; planificación, organización, gestión, administración y estudio de la viabilidad de proyectos de Diseño, entre otras. Se distinguen a su vez, las que son mayormente "sistémicas" y las separa de aquellas de carácter "interpersonal” tales como motivación por la calidad o la capacidad de iniciativa y espíritu emprendedor.

Sin embargo, la mera enumeración o identificación de posibles competencias requeridas por un estudiante de Diseño o un profesional en el inicio de su carrera, no constituye nada útil si no se ajustan a un sistema que permita su uso práctico y verificable.

En este sentido, Borja de Mozota (2003) divide las competencias de los diseñadores y de las capacidades de aplicación a una situación proyectual, en cinco categorías: Gestión del proceso (compromiso, entusiasmo, autoconfianza, orientación hacia los resultados, trabajo en equipo, mejora continua, gestión de cambio, etc.), Concepción (creatividad objetiva, capacidad técnica, capacidad para resolver un problema, observación del usuario y empatía, creación de prototipos, pensamiento conceptual, etc.), Orientación comercial (comprensión del mercado, planificación y organización de proyectos, capacidades comerciales, etc.), Perspectiva (recopilación y uso de la información, pensamiento estratégico, capacidad de colaboración, etc.) y Cualidades interpersonales (capacidad de generar relaciones, influir, flexibilizar, etc.).

Por último, existen algunos estudios que exploran necesidades muy específicas, que ciertas industrias y localidades poseen, tales como el estudio de Dziobczenski y Person (2017), quienes describen y analizan, a partir de categorías de ejecución, de conocimiento y de demostración, lo que se espera de un diseñador que labora en la industria de la publicidad en el Reino Unido.

La revisión de estos y otros antecedentes ayudaron a determinar varios puntos que definen la propuesta que a continuación se desarrolla; en el sentido de que, las habilidades del diseñador requieren indudablemente un ajuste que las proyecte como competencias útiles en un contexto futuro (NCREL y the Metiri Group, 2003); están sujetas a una realidad contextual y temporal muy particular, y su valoración e importancia varía cada vez más con mayor dinamismo (Massaguer, 2017); y que, es posible categorizarlas fácilmente dependiendo del tipo de actividad y qué tan estrictamente relacionadas se encuentran entre sí (Borja de Mozota, 2003). 
La propuesta clasificatoria que se presenta a continuación, busca organizar de forma lógica y consensuada, la gran cantidad de acciones y tareas concretas relacionadas con la enseñanza y práctica del Diseño Gráfico. Asimismo, se identifica la necesidad de ubicar diferentes niveles de dominio de estas habilidades y aunque puedan estar íntimamente relacionadas en lo que requieren, el nivel de aplicación resulta más complejo o preciso.

\section{Propuesta}

Para poder orientar los esfuerzos de los profesores de Diseño de forma eficiente es indispensable analizar con frecuencia hacia dónde se dirige la disciplina, y evaluar constantemente las destrezas asociadas con las actividades propuestas cotidianamente.

Sin embargo, lo anterior puede ser demasiado abstracto o complejo para alcanzar a verlo, por lo que, al trabajar en un modelo por competencias (o que busca reenfocarse de esta forma), no es suficiente tratar de enlistar las habilidades esperadas y dividirlas entre ideales, novedosas o complementarias. Es necesario establecer, en primer lugar, un criterio clasificatorio que tenga sentido y tenga una aplicación práctica.

En otras palabras, se requiere de un esquema de visualización que permita organizar fácilmente cualquier tarea o competencia, en un sistema de criterios clasificatorios. De esta forma, el docente será capaz de plantear nuevas tareas de logro, mejores dinámicas, conocimientos actualizados y esquemas de relación innovadores; sin dejar de lado el aspecto académico concreto y verificable del que debe acompañarse.

La propuesta podrá no ser única, pero -aunque existen valoraciones de este tipo cuando se establecen criterios de contratación, perfiles de puesto, e inclusive, en la caracterización del egreso en programas universitarios específicos- se intenta definir cada categoría y enfocar el análisis únicamente a aquellas relacionadas con un estudiante o un profesional novel del Diseño. La otra característica es que en vez de enunciar o preguntar cuáles son estas habilidades, se propone emplear en la medida de lo posible verbos en infinitivo para ubicar dichas habilidades.

Las clasificaciones que se detallan a continuación se establecieron al analizar qué características generales tienen, o deberían poseer, la generalidad de diferentes tipos de profesionistas. Por lo que, se inició la comparación buscando puntos en común para reducir al máximo el número de categorías y asignarles un nombre apropiado. Después de este análisis, se establecieron cuatro categorías finales, en las cuales (en teoría) es fácil ubicar la actividad o la habilidad en una clasificación u otra.

Cabe señalar que, se inició la propuesta con diez categorías posibles que después se redujeron a ocho. Estas fueron señaladas con una especificidad tal que muchas habilidades se ubicaban con un alto nivel de precisión pero que hizo que otras tantas, tuvieran que ubicarse en más de una categoría o, ante tanta variedad, se dudara al momento de ubicarlas en un ámbito u otro. Por lo tanto, se continuó analizando y consultando la diferenciación entre cada categoría que, finalmente, hizo que se redujera a cuatro posibilidades prácticas: estratégicas, cognitivas, instrumentales y sociales.

En primer lugar, la propuesta de clasificación no establece un orden a seguir ni tampoco prioriza cierto tipo de competencias, no se enumeran ni se hacen obvias las relaciones 
existentes. Para poder evitar que se interprete como una metodología a seguir, se propone organizarlas en un esquema elíptico que, finalmente, integra toda una serie de habilidades que debe poseer un estudiante universitario al terminar su formación académica.

Las categorías propuestas responden al desarrollo de habilidades de tipo sociocultural (determinadas por condiciones establecidas por el entorno particular, lo que genera mucha variación), otras de tipo estratégico (donde se ubican aspectos laborales, profesionales e innovadores), otras más de índole estrictamente instrumental (en este caso propias del Diseño Gráfico) y la última categoría que engloba habilidades cognitivas (en todos sus niveles de dominio y que afectan a todas las demás). Cabe señalar que, al plantearse como competencias, éstas deben ser verificables, aunque no se manifiesten claramente en los individuos, lo que a su vez da pauta para desarrollar instrumentos y sistemas específicos para cada aspecto a evaluar.

Respecto a una generalidad que ilustre el punto, se puede decir que resulta más difícil observar el desarrollo de habilidades de índole comercial en estudiantes de pedagogía, o actividades relacionadas con la cultura en la ingeniería civil. No obstante, podemos observar un desarrollo superior de las habilidades comunicativas en estudiantes de teatro o de habilidades académicas en estudiantes de música. Idealmente, los trabajadores sociales o psicólogos clínicos deberán tener un desarrollo más sensible de la empatía, así como una serie de habilidades estratégicas serán indispensables en la mercadotecnia y las relaciones públicas.

En cualquier disciplina, que acote con claridad su área de aplicación y estudio, observaremos un fuerte desarrollo de habilidades instrumentales altamente específicas pero que, en una formación universal no conviene obviar el desarrollo del resto de las habilidades, aunque sea en su nivel más básico, o al menos contar con los mecanismos para que el estudiante sea capaz de generarlos. En la Figura 1, se observa el conjunto de las distintas categorías propuestas, organizadas en el esquema elíptico antes mencionado y con tres niveles de dominio posible que parten del centro y, en la medida que se van dominando, cambian de nivel y se amplían.

Ahora bien, cualquiera de las habilidades identificadas debe permitir a su vez, distintos niveles de profundidad y permitir que se generen aprendizajes ulteriores, importante factor a considerar, ya que su contenido se puede perfeccionar, ampliar, renovar o combinar fácilmente. El enfoque por competencias no sólo contempla esto, sino que además debe indicar cómo y de qué forma se debe lograr y evaluar. El mismo modelo identifica la necesidad de generar las estrategias necesarias para seguir aprendiendo e incluso reemplazar saberes conforme se vayan haciendo obsoletos.

Esta obsolescencia será más evidente en la categoría instrumental donde el dinamismo promovido por el desarrollo tecnológico hará que las habilidades que hace (por ejemplo) 10 años eran indispensables, ahora sean irrelevantes. Lo mismo sucederá con los saberes que sean demasiado específicos, como los requeridos para utilizar sólo un programa de computación (e.g. Macromedia Flash de 1996) que invariablemente se modificarán con el tiempo y no conviene invertir demasiado en ellas. En el otro sentido, las cognitivas no cambiarán en mucho tiempo ya que será el medio o la técnica de representación lo que cambie; mientras que las socioculturales podrán variar, pero a un ritmo tan lento que será imperceptible; quizás a partir de cambios generacionales. Las estratégicas quizás no 


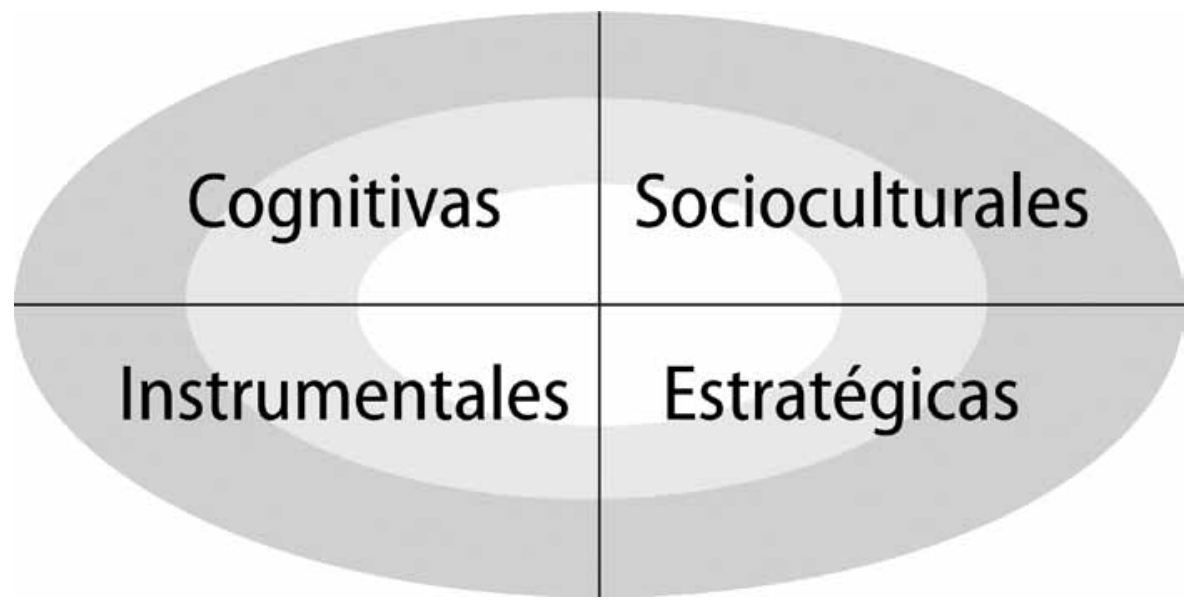

Figura 1. Sistema propuesto de clasificación de habilidades generales para el diseñador, con niveles de desarrollo básico, intermedio y avanzado. Fuente: Elaboración propia.

cambien debido al tiempo sino más bien por la situación o la industria en la que sean requeridas.

Por ejemplo, la acción de Hablar requiere del uso mínimamente adecuado de un aparato fisiológico y respiratorio que puede dominarse completamente, pero Cantar requiere de un manejo muy particular del mismo aparato en el cual intervienen otros factores instrumentales. En el mismo sentido, Hablar en francés o Contar un cuento (aspectos socioculturales) pueden entenderse como instancias de Hablar, pero no resultan tan básicas. A su vez, Exponer en clase puede entenderse como Hablar en público acerca de algo, pero requiere del uso de más recursos relacionados (volumen suficiente o lenguaje corporal apropiado) para generar una efectividad ideal, que la mera emisión oral de palabras no es capaz de lograr. Finalmente, Hacer un pitch, es decir, presentar una idea innovadora y compleja de forma concreta y convincente, con la actitud adecuada, con el contenido justo y cierto profesionalismo, en la entonación y al ritmo adecuado, implica por lo tanto una profundidad y un nivel de domino tal, que Hablar no requiere; aunque en sentido estricto, se trate de lo mismo.

Estas son las razones por las que la propuesta permite identificar distintos niveles de desarrollo que, por razones prácticas y que no comprometen al docente, se denominan básicas, intermedias y avanzadas (Ver Figura 1). En el centro del esquema se ubicarán entonces todas las habilidades obvias e indispensables, que conforme se van desarrollando y aumentando en su complejidad, se ubicarán en la banda central del círculo. De la misma forma, en el círculo externo se deberán ubicar aquellas actividades o habilidades de alta 
complejidad que requieren un alto nivel de domino que, en ocasiones, rebasarán aquellas pensadas para un estudiante de licenciatura.

En la exploración inicial de esta investigación acerca de las habilidades requeridas en Diseño, se generó una lista muy extensa y desordenada de destrezas y actitudes, muchas de las cuales no fue posible distinguir con facilidad de aquellas requeridas específicamente en un graduado (sin importar si se desempeña como profesionista independiente o como empleado en una empresa). Lo mismo podría suceder con un estudiante de posgrado que, seguramente, seguirá ampliando sus conocimientos en un área de estudio más específica y podrá seguir desarrollando sus habilidades (no sólo de tipo instrumental) en un espectro mucho mayor.

Finalmente, independiente a la relevancia que clientes, jefes, maestros y colegas brinden a cierto tipo de habilidades en contraste con el resto; la motivación y la actitud -por ejemplo- podrán ser comprobables fácilmente por medio de la observación objetiva, del trato social cotidiano y se pueden evaluar incluso con un test de personalidad, exámenes psicométricos o de conocimientos generales.

Antes de profundizar con la descripción de las categorías propuestas, cabe mencionar que el concepto holístico del esquema (y, por ende, de la propuesta), busca integrar todo un repertorio de competencias que conformarán un profesionista altamente capacitado bajo un sistema justo y en circunstancias ideales. Pero, desde un enfoque realista, cada disciplina, así como cada institución podría inclinar sus actividades formativas para definir un perfil específico que puede no contemplar o ignorar completamente otras tantas. Esto no solo es posible, sino que muchas instituciones deliberadamente se inclinan hacia alguna tendencia o se promueven como cierto tipo de escuela.

El desarrollo continuo del diseñador profesionista difícilmente se verá propiamente perfeccionado sin un equilibrio complementario de estas habilidades o de la variación de las tareas. Lo anterior hace referencia al hecho de que las habilidades mínimas indispensables para conseguir trabajo requieren ser complementadas con otras más complejas y diversas para en su momento, poder subir de puesto. Así como la habilidad instrumental seguramente se mantendrá intacta si no existe una variación en las tareas; mientras que el cambiar de contextos, empresas e industrias, ayudará a seguirlas desarrollando (en conjunto con las habilidades socioculturales) ya que requerirán la adaptación constante del individuo a distintos niveles de exigencia, diferentes esquemas culturales y psicosociales, el empleo de tecnologías distintas y/o nuevas metodologías de trabajo.

En este mismo sentido, las posibles destrezas estratégicas que el individuo pueda tener no serán suficientes si no están mejorando en eficiencia, se adaptan a nuevas situaciones, o desconoce otros mercados. Es decir, las habilidades estratégicas permitirán identificar tendencias o áreas de oportunidad que resultarán en beneficios constatables, las cuales se tendrán que complementar con aquellas de carácter cognitivo.

A su vez, cuando el alumno no posee (y como profesores no hemos hecho nada para promover) la sensibilidad suficiente para apreciar otras formas de arte, o simplemente no se interesa por relacionarse con otros grupos sociales, entender distintas culturas, aprender nuevos idiomas o tocar un instrumento musical, su desarrollo sociocultural será estacionario, y aunque sus habilidades instrumentales sean suficientes, a la larga también afectarán su desarrollo cognitivo. 


\section{Descripción de categorías y ejemplos de habilidades}

El primer problema que se presentó al iniciar a conceptualizar este esquema fue precisamente la diferenciación entre las categorías, así como el número máximo posible para que fuera práctico. Después, establecer una denominación apropiada para cada una y verificar si resulta suficientemente clara y conceptualmente amplia para que las personas que lo utilicen (profesores de Diseño principalmente) entiendan fácilmente a qué se refiere cada una y, sobre todo, si la tarea o la habilidad identificada puede ser ubicada con facilidad. Para llegar a esta propuesta, el proceso implicó comentarlo con colegas, consultarlo con académicos ajenos al Diseño y discutirlo brevemente con exalumnos, lo que hizo evidente el hecho de que la visión personal que se tiene respecto al valor o la urgencia de algunas habilidades varía enormemente. Luego, se identificaron otras habilidades que técnicamente no son consideradas como competencias (porque tienen más que ver con actitud o personalidad) pero que de igual forma son relevantes a nivel profesional y académico. A continuación, se explica a qué hace referencia cada categoría y se mencionan algunas habilidades (muchas de forma verbal) para tratar de aclarar el argumento. Otras se derivan en otras acciones que guardan cierta relación, por practicidad o por su nivel de perfeccionamiento puede que se denominen de forma independiente.

\section{Habilidades socioculturales}

Se trata del conjunto de capacidades que permiten el desarrollo de un repertorio de acciones y conductas que harán que el individuo se desenvuelva eficazmente en un ámbito social determinado. El número de variables y factores que intervienen en lo que se puede denominar como correcto, dependerá del consenso social al que se haya llegado; asimismo, la flexibilidad del criterio dependerá de la edad del individuo, la urgencia de la tarea, la claridad de las reglas establecidas, entro otros factores.

En esta categoría se incluyen habilidades básicas de tipo estrictamente social como: ser puntual (es decir, que se tiene un manejo apropiado del tiempo), ser responsable (que se cumple eficazmente con la tarea asignada), ser dedicado (lo enfocado que se esté con la tarea), comprometerse (asumir responsabilidades y llevar a término un proyecto), respetar opiniones (entender que existen distintos puntos de vista), o bien, saber aceptar críticas (aprovechar las oportunidades de retroalimentación). De la mano van otras como: ser sincero, ser honesto, ser respetuoso, que son indispensables para una convivencia social mínimamente apropiada. Otras habilidades también de tipo sociocultural, ligeramente más complejas, pueden ser: la predisposición para ayudar a los demás, interactuar apropiadamente con distintos públicos, hablar otro idioma, saber discutir de forma profesional, ser capaz de orientar, entre otras funciones deseables. Además, dependiendo del contexto se podrán valorar: tener apertura mental, vestir apropiadamente, tolerar, etc.

\section{Habilidades estratégicas}

Inicialmente, se consideró llamarles "laborales" pero la designación limitaba la acción al mero cumplimiento de las tareas, sin cuestionar necesariamente de qué forma se puede 
mejorar, qué aspectos de innovación es posible incluir o qué conviene más, si trabajar mucho o de forma inteligente. Algunos ejemplos sin ningún orden en particular pudieran ser: ceder (entender cuándo vale la pena enfrentarse o discutir), compartir información (lo que ayudará a todo el equipo de trabajo), delegar (lo que permite acelerar procesos y compartir responsabilidades) o tomar mejores decisiones (lo cual sólo puede darse con la experiencia y con la disposición a equivocarse constantemente).

De la misma forma, visualizar el panorama general (la capacidad de "alejarse" de la problemática para visualizar todos los elementos involucrados), persuadir (no sólo involucra enunciar las ventajas que algo tiene, sino lograr convencer a alguien de tomar cierta acción que inicialmente no contemplaba), adaptar (implica ser capaz de ajustar partes de algo para coincidir con características o criterios distintos), improvisar (hace referencia a la solución de una problemática con los recursos que se tienen disponibles de forma repentina), etc.

Existe una serie de destrezas que se pueden clasificar dentro del espectro sociocultural sin ningún problema, pero las habilidades comunicativas como un conjunto propio, se incluyen dentro de las estratégicas, debido al uso práctico e impacto que diferentes piezas de comunicación efectiva pueden tener, tales como: saber debatir (argumentar convincentemente una opinión informada), sintetizar (resumir información hasta su expresión más relevante), redactar (comunicar de forma escrita algo de importancia) y otras más complejas como traducir pueden tener impacto en otras como convencer y/o promover.

\section{Habilidades cognitivas}

Como se mencionó anteriormente, este conjunto de habilidades técnicamente tiene impacto en todas las demás, ya que tiene que haber un proceso de racionalización en todas las habilidades. Sin embargo, si se pueden identificar específicamente algunas como: analizar (que puede tener muchos niveles de profundidad), planear (que va desde la realización de una tarea simple con una serie de pasos predefinidos hasta la realización de un plan de negocios en una industria compleja y con un panorama ambiguo), calcular (actividades relacionadas con la numeralidad, que permitirán estimar costos, superficies, componentes, etc.), idear (pensar eficazmente en soluciones a problemáticas dadas), entre otras.

Algunas otras pueden interpretarse como claras y simples, pero no solemos ver lo complejas que pueden ser o la cantidad de tareas asociadas con las mismas, pueden relacionarse con la estrategia, pero el componente de pensamiento aplicado es lo que las hace considerables como cognitivas. Asociar, por ejemplo, se refiere a la identificación de elementos comunes entre otros disímiles y el establecimiento de una relación comparativa entre ellos gracias a una experiencia previa; hacer más eficiente (incorrectamente empleado como "eficientar"), tiene que ver con la capacidad de reducir elementos, automatizar tareas o reorganizar procesos para obtener resultados con menos posibilidad de error, empleando menos recursos o en menor tiempo; entre otras similares.

Hay otras que se distinguen a partir de su nivel de atención o si involucra acciones más específicas, que se dan naturalmente como en la diferenciación entre ver y observar, o entre oír y escuchar. Existen otra serie de habilidades interconectadas, manifestadas por la necesidad comunicativa que, al igual que las cognitivas, tienen un impacto en todas las 
demás áreas definidas. Habilidades como: redactar, resumir, parafrasear, citar, referenciar, consultar, entre muchas otras actividades van de la mano con la comunicación escrita de corte académico y con un nivel de profesionalización definido. Asimismo, habilidades como: comentar, relatar, reseñar, explicar, recomendar, exponer, declamar, a su vez, requieren de una comunicación oral que, con criterios preestablecidos y suficiente práctica, se convierten en actividades serias y valoradas.

Finalmente, existe un grupo que amerita un desarrollo de varios tipos de habilidades tales como: contextualizar (que requiere una sensibilidad sociocultural o un conocimiento histórico particular para ubicar en un entorno dado); persuadir (inducir una respuesta en alguien solamente con razones); o cautivar (ejercer irresistible influencia en el ánimo de alguien por medio de atractivo físico o moral) (RAE, 2018).

\section{Habilidades instrumentales}

Dependiendo de la disciplina en cuestión, el tipo de destrezas que habrá que desarrollar en un estudiante variarán enormemente. Aquí, se hace referencia a las actividades propias del Diseño Gráfico, mismas que pueden ser exclusivas de la disciplina (vectorizar, retocar fotografías, "logotipar", maquetar, trazar, geometrizar...), o bien, repetirse en otras actividades con diferentes acepciones tales como pintar, grabar, pegar, calcar o acomodar; que pueden llegar a ser tan específicas como difuminar o "serigrafiar".

Profundizando en uno de estos verbos, la simple acción de Cortar, puede tener una variedad enorme de interpretaciones y emplear un sinfín de técnicas distintas. Por ejemplo, la forma en cómo corta papel un diseñador (empleando una cuchilla de precisión y con una tabla para corte), guarda cierta relación con la forma en cómo penetra la piel humana el bisturí de un cirujano (donde el instrumental es similar y el proceso controlado requiere precisión y paciencia); pero, tiene poco que ver con la forma en como un niño de preescolar lo hace (quizás con unas tijeras de punta redonda) aunque el material sea el mismo. La forma en cómo lo hace una costurera con diferentes telas (sobre una mesa grande y en un solo movimiento continuo), cómo lo hace un diseñador industrial que hace una maqueta o un prototipo empleando materiales plásticos, o cómo lo hace un sous-chef (con una maestría, una rapidez y un instrumental distinto), implica definir claramente los criterios de evaluación apropiados para la misma acción. En otra situación, la acción de cortar varillas en el caso de herrero guarda más relación con cómo cortaría madera un ebanista, que poco se relaciona con lo que haría un leñador en un bosque armado de un hacha (o una sierra) aunque se trate del mismo material. Así, vemos que el simpe verbo Cortar posee una variedad instrumental y de ejecución enorme, donde la estandarización de un criterio de evaluación que no ha sido adaptado disciplinalmente, poco ayudará a definir la acción específica y lo que se pudiera designar como un resultado exitoso.

El modelo por competencias y la capacitación en un oficio se relacionan perfectamente porque el proceso de enseñanza por lo general implica, entre otras acciones, la explicación de la acción, quizás la observación previa de la misma, la prueba supervisada, la realización exitosa de la acción, para después requerir de una repetición constante hasta que dicha actividad es dominada. 
De esta forma, la propuesta presentada permite profundizar en definiciones concretas de cada acción para personalizar los criterios, normar las evaluaciones y predefinir pautas requeridas en un estudiante de Diseño. El esquema presentado permite codificar de varias formas cada una de las habilidades y establecer un seguimiento lógico que permita encauzar los esfuerzos educativos hacia acciones pertinentes, relevantes $y$, sobre todo, valiosas. Las actividades de rediseño o ajuste curricular de los programas educativos en Diseño, requieren visualizar el esquema general de las competencias que cada actividad y materia generan. Por último, este esquema busca ayudar a ubicar dichas habilidades en áreas de competencia particulares, organizarlas en distintos niveles de dominio y relacionarlas con mayor facilidad, al entender que muchos de los requisitos cognitivos e instrumentales guardan mucha relación.

\section{Comentarios finales}

En este documento se plantea de forma general una serie de situaciones relacionadas con la formación universitaria de un diseñador y contempla, aunque inicialmente de forma superficial, las necesidades que un profesionista novel tendrá en su carrera. También, se indicó la preocupación generalizada de muchas instituciones y profesores al trasladar los programas de estudio hacia un Modelo Curricular por Competencias donde es de vital importancia el establecimiento de criterios claros y hasta cierto punto consensados de cómo formar diseñadores, cómo establecer las pautas para su evaluación objetiva y práctica y, sobre todo, qué nuevas acciones, saberes y criterios se deben incluir en la mezcla para prepararlos mejor ante situaciones o problemas futuros.

De aquí pueden partir otra serie de estudios y propuestas, quizás más específicas o con aplicaciones dirigidas a un público o una institución en particular, que permitirán definir claramente qué habilidades se pretende desarrollar con determinada tarea, verificar si el orden es el apropiado e incluso ayudar a cuestionar su relevancia. Con respecto a la posible personalización de la propuesta, hay que considerar la relativa facilidad con la que se puede integrar el modelo elíptico aquí descrito y hacerlo corresponder con todas las temáticas, tareas, materias y actividades de un programa de estudios particular, y de esta forma determinar con precisión qué se está enseñando, para qué, de qué forma y en qué orden; mientras que se puede visualizar todo fácilmente.

El esquema elíptico planteado puede tener una correlación numérica con el tipo de habilidad y el nivel de dominio que permita enlistar, por ejemplo, las acciones a tomar dentro de una clase o en una materia en específico, codificar fácilmente las habilidades y evaluar su dominio en los estudiantes. Las mallas curriculares y, particularmente, las cartas descriptivas de las materias también pueden incluir este diagrama para ubicar visualmente qué tipo de contenidos posee y en qué contexto se ubica la tarea. Al relacionarlo con el número de horas teóricas o prácticas de cada una de las materias también se pueden codificar o enumerar con relativa sencillez.

Para las instituciones de educación superior que estén en proceso de rediseño o ajuste curricular, esta propuesta puede resultar también atractiva para ayudar a todos los académicos involucrados (que en ocasiones no son diseñadores de profesión) a entender el 
orden, la profundidad y la relación entre las materias, facilitando la organización de los contenidos, o bien, la distribución de créditos entre distintas áreas.

Finalmente, esta propuesta se pone principalmente a consideración de los académicos del Diseño pero existe un público enorme que difícilmente se ha tomado en cuenta conformado de egresados y graduados, exalumnos en proceso de titulación y sobre todo, de profesionistas noveles, quienes en sus nuevas problemáticas cotidianas empiezan a ubicar con mayor claridad, el tipo de tareas o habilidades que debieron desarrollar en su formación y que ahora les están haciendo falta.

Aunque el mecanismo de integración de esta información puede ser complicada y en muchos no será posible ni siquiera obtenerla, no hemos sido capaces de apreciar lo valioso que podría ser este input en la educación del Diseño, la mejora institucional y lo útil que a la larga sería para nuestra discilpina contar con una retroalimentación constante. Muchos de estos profesionistas noveles seguramente podrían contribuir de forma activa y concreta a mejorar -por medio de instrumentos de evaluación precisos, nuevas propuestas metodológicas o temáticas realmente actualizadas- la educación superior del Diseño.

\title{
Lista de Referencias Bibliográficas
}

Borja de Mozota, B. (2003). Design Management: Using Design to Build Brand Value and Corporate Innovation. New York: Allworth Press.

Dirección General de Educación Superior para Profesionales de la Educación (DGESPE). (s/f). Enfoque centrado en competencias. Recuperado de http://www.dgespe.sep.gob.mx/ reforma_curricular/planes/lepri/plan_de_estudios/enfoque_centrado_competencias

Dziobczenski, P. R. N. \& Person, O. (2017). Graphic designer wanted: A document analysis of the described skill set of graphic designers in job advertisements from the United Kingdom. International Journal of Design, 11(2), 41-55.

Massaguer, L. (2017). Relació entre les competències académiques i les professionals en el perfil de dissenyador/a gràfic/a. Grafica. Documents de diseeny gráfic, 5(10), 95-103. http://dx.doi.org/10.5565/rev/grafica.86

North Central Regional Educational Laboratory (NCREL) y the Metiri Group. (2003). EnGauge 21st Century Skills. Literacy in the Digital Age. Recuperado de http://pict.sdsu. edu/engauge21st.pdf

Real Academia Española. (2017). Diccionario de la lengua española. Recuperado de http:// www.rae.es/rae.html

\begin{abstract}
After reviewing previous works related to competency assessment, a scheme is proposed that locates in clearly defined categories the broad repertoire of skills associated with the professional training of a graphic designer. The main intention is to organize specific competences that allow different levels of development. The proposal can be used on a daily basis or in institutional projects to redesign curricula or study plans as it allows
\end{abstract}


to visualize with relative ease the impact and relevance of multiple academic activities typically associated with graphic design.

Keywords: Design - skills - competences - higher education - profession.

Resumo: Após a revisão de antecedentes relacionados à avaliação de competências, propõe-se um esquema que coloque em categorias claramente definidas o amplo repertório de habilidades associadas à formação profissional de um designer gráfico. A principal intenção é organizar competências específicas que permitam diferentes níveis de desenvolvimento. A proposta pode ser usada diariamente ou em projetos institucionais para redesenhar currículos ou ajustes curriculares, pois permite visualizar com relativa facilidade o impacto e da relevância de várias atividades acadêmicas tipicamente associado com design gráfico.

Palavras chave: Design - habilidades - competências - ensino superior - profissão.

[Las traducciones de los abstracts fueron supervisadas por el autor de cada artículo] 